\title{
Effects of Target Gas in Collision-Induced Dissociation Using a Double Quadrupole Mass Spectrometer and Radiofrequency Glow Discharge
}

\author{
Yuan Mei, Douglas C. Duckworth,* Paula R. Cable, and \\ R. Kenneth Marcus \\ Department of Chemistry, How ard Hunter Chemical Laboratories, Clemson University, Clemson, South \\ Carolina, USA
}

\begin{abstract}
Collision-induced dissociation (CID) of polyatomic ions sampled from an rf-powered glow discharge is examined by using three target gases including atomic ( $\mathrm{Ar}$ and $\mathrm{Xe}$ ) and molecular species $\left(\mathrm{N}_{2}\right)$. Collisions with these targets in the first quadrupole of the double quadrupole system result in the loss of discharge species by dissociation, symmetric and asymmetric charge exchange, and scattering, each to varying degrees. These processes are seen to be a function of the relative mass, size, and ionization potentials of the target species, as well as the collision center-of-mass energies. In light of the comparisons, xenon appears to be the best collision target for both CID and charge exchange because of its relatively low ionization potential and high dissociation efficiency of polyatomic species. Evidence for both symmetric and asymmetric charge exchange is presented for Ar and Xe target gases. (J Am Soc Mass Spectrom 1994, 5, 845-851)
\end{abstract}

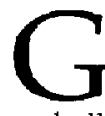
low discharge mass spectrometry (GDMS) is a sensitive technique for the direct elemental analysis of conducting solids such as metals and alloys, and continues to grow in both the number of practitioners and the range of applications [1,2]. Primary limitations of the GDMS technique include the need for electrically conductive samples and the presence of isobaric interferences. In this laboratory, radiofrequency- ( $\mathrm{rf}$ ) powered glow discharges have been developed to eliminate the need for electrical conductivity in samples, which allows the direct analysis of nonconducting materials such as glasses and specialty ceramics $[3,4]$. Although the ability to analyze nonconductive solids is a true advantage of the $\mathrm{rf}$ sources, the presence of polyatomic species is often enhanced in the mass spectra derived from the device described here. The increased occurrence of such species is likely due to source geometry-dependent lower operating pressures $(\sim 150$ mtorr) relative to the dc-powered glow discharge (1-3 torr). (Optimum source operating pressures are inversely related to ion source volumes, and this particular system employs a relatively large chamber.) Harrison and co-workers [5] found that polyatomic species tend to dissociate

Address reprint requests to Dr. R. Kenneth Marcus, Department of Chemistry, Howard L. Hunter Chemical Laboratories, Clemson University, Clemson, SC 29634-1905.

* Present address: Chemical and Analytical Sciences Division, Oak Ridge National Laboratory, Oak Ridge, TN 37831-6375. through gas-phase collisions within the discharge at higher source pressures, which decreases the relative abundance of these species in the plasma. Also it seems that the rf glow discharge is more efficient than the dc-powered sources in electron impact ionization of the discharge gas species, including residual gases, because of the oscillatory nature of the rf plasma sheaths [6]. Hence, if discharges, in general, tend to be more prone to interferences from residual gases and their adducts.

The isobaric interferences encountered in GDMS consist of interelement interferences, discharge gas species $\left(\mathrm{Ar}^{+}, \mathrm{Ar}_{2}^{+}\right)$, atomic and molecular residual gases $\left(\mathrm{H}_{2}^{+}, \mathrm{H}^{+}, \mathrm{N}_{2}^{+}, \mathrm{N}_{2} \mathrm{H}^{+}, \mathrm{O}_{2}^{+}, \mathrm{O}^{+}\right.$, etc.), analyte ${ }^{-}$ discharge gas complexes $\left(\mathrm{MAr}^{+}\right)$, analyte dimers and trimers $\left(\mathrm{M}_{2}^{+}, \mathrm{M}_{3}^{+}\right)$, analyte-residual gas complexes $\left(\mathrm{MO}^{+}, \mathrm{MH}^{+}, \mathrm{MN}^{+}\right.$, etc.), and multiply charged species $\left(\mathrm{M}^{2+}, \mathrm{Ar}^{2+}, \mathrm{Ar}^{3+}\right.$, etc. $)$. The use of high purity discharge gas $(99.999 \%)$, good vacuum practices, and judicious choice of discharge conditions are effective means of reducing polyatomic interferences.

Another method of reducing polyatomic isobars is through collision-induced dissociation (CID) with gas atoms or molecules in a specified collision cell. The preferential loss of polyatomic species through gasphase collisions in both double [7] and triple [8] quadrupole mass spectrometers has been demonstrated for ions sampled from an inductively coupled plasma and a dc-powered glow discharge, respec- 
tively. In a related work, Duckworth and Marcus [9] used a double quadrupole mass spectrometer to sample ions produced in an rf-powered glow discharge that employed the first quadrupole as a collision cell with subsequent mass filtering in the second quadrupole. Argon was employed as the collision target, which affected the preferential loss $(>90 \%)$ of most interfering species relative to the analyte. Transmission of monoatomic analyte ions was fairly efficient under CID conditions, with a $20-30 \%$ beam intensity retention. As an extension of that work, a comparison of three collision target gases was made and is presented here. Both atomic $(\mathrm{Ar}, \mathrm{Xe})$ and molecular gases

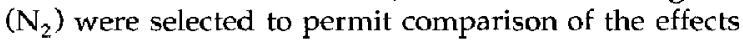
of the different masses, sizes (degree of vibrational freedom), and ionization potentials of these gases on the extent of dissociation, scattering, and charge exchange of various discharge species.

\section{Experimental}

The ion source used in this study consisted of a $6.98-\mathrm{cm}$ six-way-cross ion volume and an rf-powered direct insertion probe designed for glow discharge mass spectrometry [4]. The rf potential was applied to the sample, which was mounted on the end of an electrical feedthrough that extended through the center of the probe. The probe body served as a grounded shield of the rf power. The fundamentals of rf glow discharge operation have been presented elsewhere $[3,6]$. Here, a 0.476-cm-dia. $X \sim 1$-cm-thick stock copper pellet served as the sample throughout the study. The probe was inserted through a vacuum interlock assembly into a stainless steel six-way cross that housed the discharge. The plasma was powered by a $13.56-\mathrm{MHz}$ sinusoidal generator through its associated impedance matching network (Models RF5S and AM5, respectively; RF Power Products, Inc., Marlton, NJ). High purity (99.999\%) argon was used as the discharge gas in all cases and its pressure was monitored by means of a thermocouple gauge (Model DV-23, Teledyne Hastings-Raydist, Hampton, VA). Discharge parameters were set to promote the formation of polyatomic species sampled from the discharge [rf power $=34 \mathrm{~W}$ ( $0 \mathrm{~W}$ reflected); discharge gas pressure $=140 \mathrm{mtorr}]$.

Ions of the sputtered species, discharge gas, and polyatomic ions diffuse through the 1 -mm-diameter orifice in the center of the grounded sampling cone into a differentially pumped region. The ions were then extracted through a skimmer cone into an acceleration-offset-deceleration lens system to the first quadrupole (Q1), similar to the setup described by $\mathrm{Hu}$ and Houk [10]. The mass spectrometer consisted of two sets of 0.953 -cm-diameter Extrel (Pittsburgh, PA) quadrupoles that served as the collision cell (Q1, operating in the rf-only mode) and the mass analyzer (Q2). The first quadrupole was housed in a stainless steel "can" with a 6.35-mm-diameter entrance aperture and a $9.52-\mathrm{mm}$-diameter exit as defined by a leaky dielec- tric lens component. Mass-to-charge filtered ions were detected on a conversion dynode-channeltron multiplier pair. The mass spectrometer lens potentials were controlled by Extrel C-50 components whereas intensity measurements and sweep functions were performed with a Labview 2 (National Instruments, Austin, TX) program written in this laboratory for a Macintosh IIsi microcomputer that employs a NBIO$16 \mathrm{X}$ multifunction interface board.

High purity target gases (99.999\%) used in this study ( $\mathrm{Ar}, \mathrm{Xe}$, and $\mathrm{N}_{2}$; Spectra Gases, Newark, NJ) were introduced into the collision cell through a controlled leak valve. The actual pressure in the collision cell could not be monitored directly, and was therefore related to the pressure in the surrounding chamber, measured by a gas ionization gauge and controller (Models 307 and 274012, respectively; Granville Phillips Co., Boulder, $\mathrm{CO}$ ). The pressure for each target gas was corrected by using the gauge manufacturer's relative sensitivity factors. Signal intensities of the following polyatomic and atomic species were monitored as a function of the collision energy and target gas pressure for each of the gases mentioned above: $\mathrm{Ar}^{2+}, \mathrm{Ar}^{+}$, $\mathrm{ArH}^{+}, \mathrm{Cu}^{+}, \mathrm{Ar}_{2}^{+}, \mathrm{CuAr}^{+}$, and $\mathrm{Cu}_{2}^{+}$.

\section{Results and Discussion}

The main objective of dissociating polyatomic species in the gas phase is to affect the preferential loss of polyatomic ions with respect to the monatomic analyte species of interest. Primary mechanisms of parent ion loss are (1) collision-induced (or activated) dissociation, (2) symmetric or asymmetric charge exchange, and (3) elastic scattering by target gas atoms or molecules. Atomic ions are also lost by elastic scattering and charge exchange, provided the latter is energetically favorable.

\section{Optimization of Q1 Offset Potentials}

One of the main factors that affects the efficiency of CID is the collision energy. Collision energy is classically related as the center-of-mass energy [11]

$$
E_{\mathrm{cm}}=\frac{M_{\mathrm{t}}}{\left(M_{\mathrm{t}}+M_{\mathrm{p}}\right)}\left(E_{\text {mean }}+\mathrm{Q} 1\right)
$$

where $M_{t}$ and $M_{p}$ are the masses of the target gas and parent ion, respectively. $E_{\text {mean }}$ is the mean kinetic energy of the ions of interest as they enter the collision cell, and Q1 is the dc bias potential on the collision cell. In most tandem mass spectrometry applications, the $E_{\text {mean }}$ term is not included because ions that exit in the source region have nominal kinetic energies. In the rf glow discharge applications, mean kinetic energies are dependent on the plasma conditions (i.e., plasma potential) and hence do not vary significantly as long as the same discharge power and pressure are repro- 
duced among all experiments. In this study, $E_{\text {mean }}$ values were calculated by using the "fall-off potential" method described by Chambers and Hieftje [12], wherein retarding grids were employed to energyanalyze the ion population sampled from an inductively coupled plasma. Olivares and Houk [13] illustrated the utility of quadrupole rod bias as a means of ion energy analysis in inductively coupled plasma mass spectrometry (ICP-MS). Fall-off potentials were derived in the work described here by use of the pole bias of the rf-only quadrupole, because they would be expected to be more representative of the ion entering the collision environment than the retarding grid approach. A detailed description of this approach to measure and calculate kinetic energies of ions extracted from an if glow discharge will be published in a separate article. Once $E_{\text {mear }}$ is determined, the $E_{\mathrm{cm}}$ for a given ion-target gas combination becomes dependent primarily on $\mathrm{Q} 1$, the potential offset of the collision cell with respect to the ion source potential (ground in this case). Table 1 lists a set of center-of-mass energies for collisions between various ions and $\mathrm{Ar}$ under a series of $Q 1$ settings by using the determined $E_{\text {mean }}$ value of $8 \mathrm{eV}$ [the determined kinetic energies were uniform $(< \pm 1 \mathrm{eV})$ across the mass range studied here]. The primary goal of this phase of the study was to optimize the $Q 1$ bias for all three target gases to achieve dissociation of a maximum amount of molecular species while preserving the atomic ions.

It is important to point out that there are certain limitations to this optimization process because $E_{\mathrm{cm}}$ and $Q 1$ cannot be simultaneously held constant for all ions. As can be seen from Table $1, E_{\mathrm{cm}}$ values differ as a function of mass under the same Q1 setting. By the same token, if the $E_{\mathrm{cm}}$ is kept constant for all ions, Q1 must be varied such that the value is different for each ion. Thus the ion transmission (throughput) characteristics of the first quadrupole are changed and hence a comparison of the actual ion signal intensities obtained under different $Q 1$ settings is difficult. Therefore, we chose to use the ion signal ratio $\ln \left(I_{0} / I\right)$ (the significance of which will be described subsequently) to evaluate the effect of Q1 on the CID effectiveness, where $I_{0}$ and $I$ are signal intensities without and with the CID target gas present, respectively, Q1 was varied

Table 1. Center-of-mass energy $\left(E_{\mathrm{cm}}\right)$ dependence on collision cell bias (Q1) for the collision of

various ions with $\operatorname{Ar}\left(E_{\text {mean }}=8 \mathrm{eV}\right)$

\begin{tabular}{|c|c|c|c|c|c|}
\hline \multirow[b]{2}{*}{$\operatorname{lon}$} & \multicolumn{5}{|c|}{$E_{\mathrm{cm}}\{\mathrm{eV})$ at various $\mathrm{Q} 1(\mathrm{~V})$} \\
\hline & $\mathrm{a} 1=0$ & $\mathrm{a} \mathbf{1}=-\mathbf{8}$ & $\mathrm{Q} 1=-17$ & $\mathrm{Q1}=-25$ & $\alpha 1=-34$ \\
\hline $\mathrm{Ar}^{2+}$ & 5.3 & 16.0 & 28.0 & 38.6 & 50.6 \\
\hline $\mathrm{Ar}^{+}$ & 4.0 & 8.0 & 12.5 & 16.5 & 21.0 \\
\hline $\mathrm{ArH}^{+}$ & 4.0 & 8.0 & 12.5 & 16.3 & 20.7 \\
\hline $\mathrm{Ar}_{2}^{+}$ & 2.7 & 5.3 & 8.3 & 11.0 & 14.0 \\
\hline $\mathrm{Cu}^{+}$ & 3.1 & 6.2 & 9.7 & 12.8 & 16.3 \\
\hline CuAr ${ }^{+}$ & 2.2 & 4.5 & 7.0 & 9.2 & 11.8 \\
\hline $\mathrm{Cu}_{2}^{+}$ & 2.0 & 3.8 & 6.0 & 8.0 & 10.1 \\
\hline
\end{tabular}

within a certain range that did not deteriorate the ion transmission and optimized at different values for the different target gases. By using $\mathrm{Ar}$ as the target gas, the signal attenuation factors were measured for $\mathrm{Ar}^{+}$, $\mathrm{Ar}_{2}^{+}, \mathrm{Cu}^{+}$, and $\mathrm{Cu}_{2}^{+}$while $\mathrm{Q} 1$ was varied between $\sim-40$ and $10 \mathrm{~V}$ (corresponding to center-of-mass energies of 11.5 and $0.7 \mathrm{eV}$ for $\mathrm{Cu}_{2}^{+}$). As shown in Figure 1, the highest loss ratio for $\mathrm{Cu}_{2}^{+}$was obtained at a bias of $\sim-8 \mathrm{~V}$. This collision bias value also yielded the largest difference between the values for $\mathrm{Cu}^{+}$and $\mathrm{Cu}_{2}^{+}$. Based on the previous discussion, the most optimal collision cell bias potential for CID studies when Ar was the target gas is $Q 1=-8 \mathrm{~V}$, which corresponds to an $E_{\mathrm{cm}}$ of $3.8 \mathrm{eV}$ for $\mathrm{Cu}_{2}^{+}$. Similar studies were also carried out with $\mathrm{Xe}$ and $\mathrm{N}_{2}$. The best Q1 values for each gas along with the corresponding $E_{\mathrm{cm}}$ values for all ions of interest are listed in Table 2. These Q1 settings are used throughout the remainder of this study for the respective target gases.

\section{Comparison of CID Efficiencies among Target Gases}

Another main factor that affects CID efficiency is the target gas number density (pressure), which can be related to the reduced ion beam intensity ( $I$ ) by a Beer's law analogy as

$$
I=I_{0} e^{-n \sigma L}
$$

where $I_{0}$ is the initial intensity (without collision gas), $n$ is the number density (pressure) of the target gas, $\sigma$ is the cross section for loss, and $L$ is the path length of the ion trajectory. Higher number densities increase collision rates and therefore increase the CID efficiency. Unfortunately, the attenuation of the beam due to ion loss caused by elastic scattering also increases with the gas pressure. Thus, a trade-off exists between the atomic losses because of scattering and the polyatomic losses because of CID as the collision pressure is increased. The cross section for loss, $\sigma$, encompasses many possible factors that affect ion loss including collision energy (which is proportional to the reduced mass of the collision partners), size (geometric cross

Table 2. Center-of-mass energies for various ion-gas collisions under optimized $Q^{1}$ bias

$\left(E_{\text {me an }}=8 \mathrm{eV}\right)$

\begin{tabular}{lccc}
\hline & \multicolumn{3}{c}{$E_{\mathrm{cm}}(\mathrm{eV})$} \\
\cline { 2 - 3 } $\operatorname{lon}$ & $(\mathrm{Ar}=-8 \mathrm{~V})$ & $(\mathrm{X} 1=1 \mathrm{~V})$ & $(\mathrm{N} 1=-10 \mathrm{~V})$ \\
\hline $\mathrm{Ar}^{2+}$ & 16.0 & 6.0 & 10.5 \\
$\mathrm{Ar}^{+}$ & $\mathbf{8 . 0}$ & 5.4 & 7.4 \\
$\mathrm{ArH}^{+}$ & $\mathbf{8 . 0}$ & 5.3 & 7.3 \\
$\mathrm{Ar}_{2}^{+}$ & 5.3 & 4.4 & 4.7 \\
$\mathrm{Cu}^{+}$ & 6.2 & 4.7 & 5.5 \\
$\mathrm{CuAr}^{+}$ & 4.5 & 3.9 & 3.8 \\
$\mathrm{Cu}_{2}^{+}$ & 3.8 & 3.6 & 3.3 \\
\hline
\end{tabular}




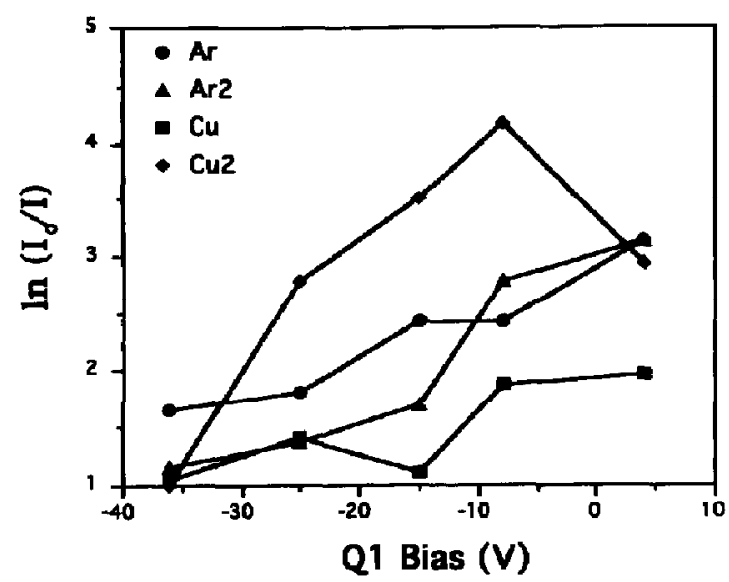

Figure 1. Signal attenuation of $\mathrm{Ar}^{+}, \mathrm{Ar}_{2}^{+}, \mathrm{Cu}^{+}$, and $\mathrm{Cu}_{2}^{+}$as functions of the collision cell bias Q1 with Ar as the target gas (rear chamber pressure $\sim 40 \times 10^{-5}$ torr).

section and vibrational degrees of freedom), and relative ionization potentials. Characteristics of the target gases chosen for this study are given in Table 3 and represent a variety of combinations of mass, size (van der Waals geometric cross sections), and ionization potentials (IPs) for both atomic and molecular species. Employing gaseous targets with a range of these characteristics provides a basis for comparison of the various factors that affect ion loss processes.

For each of the target gases employed, the pole bias (Q1) of the collision cell was first set to the value corresponding to the previously determined appropriate center-of-mass energy. Signal intensity data were then collected for the monitored species across a pressure range. Actual pressures in the collision cell are expected to be on the order of 1 mtorr. Figure 2 illustrates the extent of signal attenuation for various discharge species, both atomic and polyatomic, as a function of collision cell pressure using Ar as the collision target. Here, the extent of signal attenuation is expressed in the natural logarithm (In) of $I_{0} / I$ to establish a linear or pseudolinear relationship with pressure that is proportional to the parameter $n$ in eq 2. The slope of the $\ln \left(I_{0} / I\right)$ versus pressure curve is indicative of the cross section for loss of the specific ionic species. We will refer to this logarithmic function as the attenuation factor. It is important to note that

Table 3. Characteristics of target gas that affect collision processes

\begin{tabular}{lrrcc}
\hline Gas & Mass (u) & $\begin{array}{c}\text { 1st IP } \\
(\mathrm{eV})\end{array}$ & $\begin{array}{c}\text { 2nd IP } \\
(\mathrm{eV})\end{array}$ & $\begin{array}{c}\text { Approximate } \\
\text { size }\left(\AA^{2}\right)^{\mathbf{a}}\end{array}$ \\
\hline \hline $\mathrm{Ar}$ & 39.95 & 15.8 & 27.6 & 11.10 \\
$\mathrm{Xe}$ & 131.29 & 12.1 & 21.21 & 14.65 \\
$\mathrm{~N}_{2}$ & 28.01 & 15.6 & & 14.14 \\
\hline
\end{tabular}

'Calculated from van der Waals' radii (CRC Handbook of Chem istry and Physics, 57th ed.; CRC Press: Cleveland, OH, 1975).

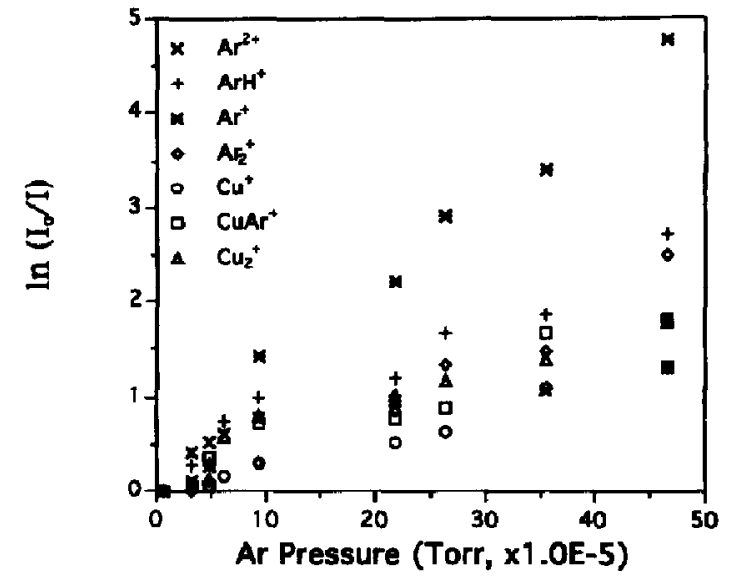

Figure 2. Attenuation factors for discharge ions as a function of Ar target gas pressure $(\mathrm{Q} 1=-8 \mathrm{~V})$.

the analyte atomic species $\left(\mathrm{Cu}^{+}\right.$in this case) is least removed whereas the unwanted polyatomic ions are attenuated to higher degrees. In Figure 2, the highest attenuation factor is found for $\mathrm{Ar}^{+}$. The reason for this phenomenon is a symmetric charge exchange reaction, which will be discussed in a subsequent section.

Similar experiments were also performed using $X_{e}$ and $\mathrm{N}_{2}$ as the target gases, and the data are shown in Figures 3 and 4 . In the case of Xe (Figure 3 ), only the four ion species of greatest interest were chosen for monitoring because of limited data collection time for the small supply of $\mathrm{Xe}$ gas on hand. Nevertheless, a comparison of the $\ln \left(I_{0} / I\right)$ versus pressure plots among the three target gases shows that within the same target pressure range, $X_{e}$ yielded the highest degree of attenuation for copper dimer and copper argide ions. This finding is likely due to the heavier mass of the $\mathrm{Xe}$ atoms. This mass-related phenomenon is especially obvious in the loss of $\mathrm{Cu}^{+}$because of scattering, which

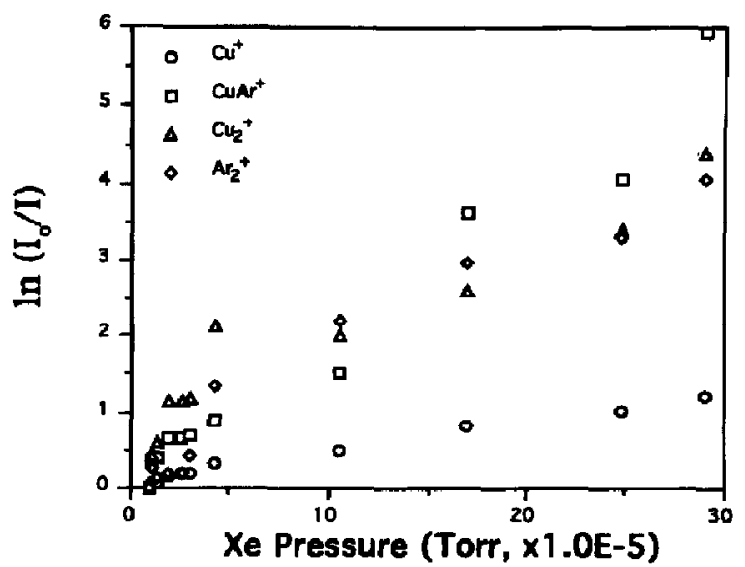

Figure 3. Attenuation factors for discharge ions as a function of Xe target gas pressure $(\mathrm{Q} 1=+1 \mathrm{~V})$. 


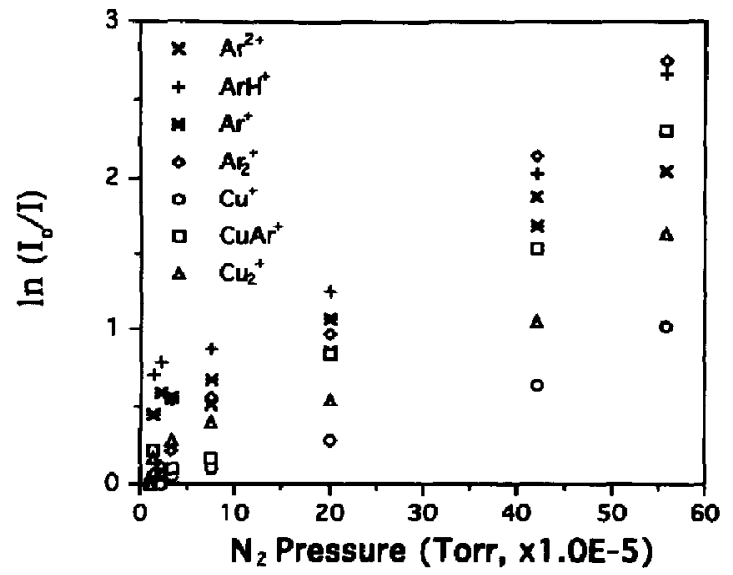

Figure 4. Attenuation factors for discharge ions as a function of $\mathrm{N}_{2}$ target gas pressure $(\mathrm{Q} 1=-10 \mathrm{~V})$.

is $\sim 2 \times$ greater for $\mathrm{Xe}$ [with $\ln \left(I_{0} / I\right)=1.0$ at $2.8 \times$ $10^{-5}$ torr] than for Ar [with $\ln \left(I_{0} / I\right)=0.6$ at $2.6 \times 10^{-5}$ torr]. Alternatively, the attenuation factors observed for each of the molecular species at the highest pressure depicted here represent over $98 \%$ reductions relative to the non-CID cases. At pressures above those depicted in the plot, these signals are below the noise level of the instrument, which suggests that reduction of the background noise levels (inherent to the current system) would yield higher observed CID efficiencies in the same pressure regime.

The attenuation characteristics for the $\mathrm{N}_{2}$ target gas are quite similar to those of Ar except for the attenuation of the $\mathrm{Ar}^{+}$ions. (The fact that the $\mathrm{Ar}^{+}$signal is not attenuated to a greater extent is somewhat surprising, and will be discussed in a subsequent section.) The near linear relationship between $\ln \left(I_{0} / I\right)$ and the target pressure appears to be better defined in Figure 4 , especially at the higher pressures. A closer examination of the extent of attenuation among the molecular species in Figure 4 reveals a decreasing order of $\mathrm{Ar}_{2}^{+}>$ $\mathrm{ArH}^{+}>\mathrm{CuAr}^{+}>\mathrm{Cu}_{2}^{+}$. Comparison of this order to the molecular dissociation energies determined experimentally by others may prove enlightening. For example, Moseley et al. [14] determined a 1.33-eV dissociation energy for $\mathrm{Ar}_{2}^{+}$, whereas calculation by Bauschlicher et al. [15] generated a value of $0.379 \mathrm{eV}$ for $\mathrm{CuAr}^{+}$. [The latter (theoretical) value would seem reasonable based on photodissociation studies by Lessen and Brucat [16, 17] of a number of transition metal-argon diatomic ions.] The bond dissociation energy for $\mathrm{Cu}_{2}^{+}$has been calculated to be $\sim 1.8 \mathrm{eV}$ [18]. Thermodynamic calculation $\left(\Delta H_{0}^{f}\right)$ of the dissociation energy of $\mathrm{ArH}^{+}$has yielded a value of $\sim 1.6 \mathrm{eV}$. These values indicate a $\mathrm{CuAr}^{+}>\mathrm{Ar}_{2}^{+}>\mathrm{ArH}^{+}>\mathrm{Cu}_{2}^{+}$order of dissociation efficiency, which is in general agreement with the previous order observed in our study, with the exception of $\mathrm{CuAr}^{+}$. Further comparison of the degrees of attenuation obtained with our double quadrupole system to those appearing elsewhere will be a point of keen interest in future studies.

For multiple quadrupole approaches to be analytically useful in GDMS, excessive losses of monatomic analyte ions in the CID processes must be minimized. One indication of the effectiveness and usefulness of CID is the fractional changes in the signal intensity of an unwanted species (e.g., CuAr) to that of a corresponding analyte atom (Cu), which is mathematically expressed as the $\mathrm{CuAr}^{+}-\mathrm{Cu}^{+}$ratio. A comparison of these fractional signal changes among the three target gases is illustrated in Figure 5. Here, to have the same reference point for comparison, the respective ratios have been normalized to their values obtained without the presence of each target gas. As seen in Figure 5, Xe is clearly superior to the other two gases in preferentially removing the argide over the atomic analyte, with the normalized $\mathrm{CuAr}{ }^{+}-\mathrm{Cu}^{+}$ratio approaching 0.002 at higher pressure. $N_{2}$ also demorstrates modestly high removal efficiency of the argide ions with respect to the analyte; this is probably because of its greater retention of the atomic ion species. Ar does not appear to remove the argide jons preferentially over the analyte ions, and this hinders it from being analytically viable as a CID gas for GDMS.

\section{Assessment of Charge Exchange in the Ion Loss Process}

As mentioned in the previous discussion, one of the primary mechanisms of ion losses in the "collision cell" is believed to be charge exchange reactions, which include both symmetric and asymmetric charge exchange that are known to occur in low energy (10-50eV) collisions [19]. These reactions are expressed by the following equations:

$$
\begin{aligned}
& A_{1}^{+}+A_{2} \rightarrow A_{1}+A_{2}^{+} \quad \text { (symmetric) } \\
& A_{1}^{+}+B_{1} \rightarrow A_{1}+B_{1}^{\prime} \quad \text { (asymmetric) }
\end{aligned}
$$

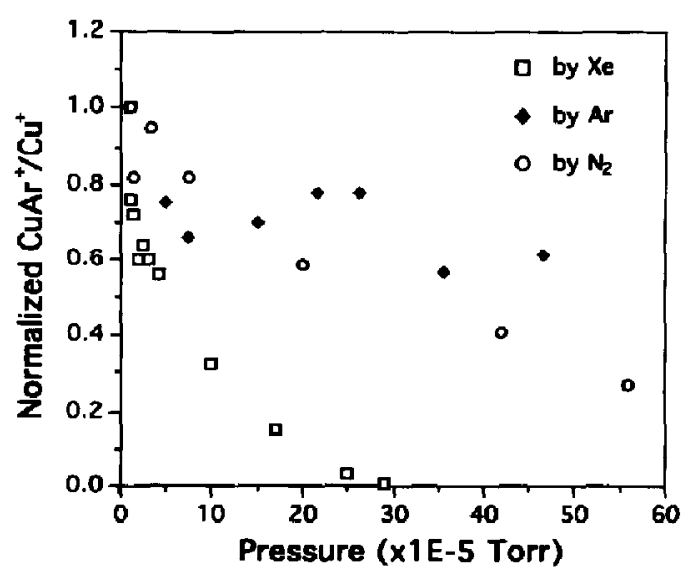

Figure 5. Effect of target gas pressure and identity on the relative signal of copper argide ions to that of monatomic copper $\left(\mathrm{Q1} 1_{\mathrm{Ar}}=-8 \mathrm{~V}, \mathrm{Q1}_{\mathrm{Xe}}=+1 \mathrm{~V}\right.$, and $\left.\mathrm{Q1} 1_{\mathrm{N}_{2}}=-10 \mathrm{~V}\right)$. 
where the incident ions $\left(\mathrm{A}_{1}^{+}\right)$have kinetic energies of approximately $E_{\mathrm{lab}}$ (i.e., $E_{\text {mean }}+\mathrm{Q} 1$ ) and the resultant ions are observed at lower kinetic energies related to their formation environment (i.e., the $\mathrm{Q} 1$ bias). The very efficient symmetric charge exchange for the $\mathrm{Ar}^{+}$-Ar pair has been verified at low energies in drift tube experiments by Hergerburg and coworkers [20]. We believe the same process contributed to the high degree of $\mathrm{Ar}^{+}$signal attenuation observed in Figure 2, and is further evidenced by the following study.

In this experiment, the signal attenuation factors were measured for $\mathrm{Ar}^{+}, \mathrm{Cu}^{+}$, and $\mathrm{Ar}_{2}^{+}$under a series of bias voltages on the mass analyzer quadrupole with $\mathrm{Ar}$ as the target gas (Figure 6). As the mass analyzer pole bias was incremented from positive to negative values (with the collision cell bias maintained at -8 $v)$, the energy range of ions that could be passed through the second quadrupole was changed. In other words, low energy (charge exchange products) ions are more readily passed through the mass analyzer by more negative bias voltages, whereas only higher energy (plasma) ions can overcome the more positive pole biases and be detected. In Figure 6 , the $\ln \left(I_{0} / I\right)$ ratio for $\mathrm{Ar}^{+}$decreased by a total of $70 \%$ as the pole bias was changed from 8 to $-8 \mathrm{~V}$. This finding suggests that $I$ was increased during this process by the contribution from low energy $\mathrm{Ar}^{+}$ions generated in the symmetric charge exchange reactions. Similar trends, but to a much lesser degree, also can be observed for the $\mathrm{Ar}_{2}^{+}$ions in Figure 6, which suggests a possible asymmetric charge exchange between $\mathrm{Ar}_{2}^{+}$ and Ar. The attenuation factor for $\mathrm{Cu}^{+}$remains fairly stable throughout the course of pole bias change, which indicates that no charge exchange reaction occurred between $\mathrm{Cu}^{+}$and $\mathrm{Ar}$ during the collision process.

Different from the case of symmetric charge exchange, the possibility of asymmetric charge exchange goes far beyond the considerations of energy level proximity. The attenuation data of $\mathrm{Ar}^{+}$in the collision with $\mathrm{N}_{2}$ is a very good example. The asymmetric

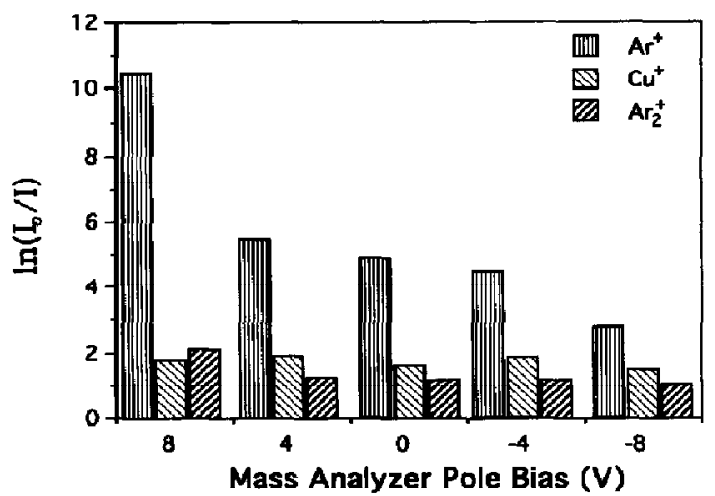

Figure 6. Signal attenuation factors as a function of the analyzer quadrupole $(\mathrm{Q} 2)$ bias for ions with different propensities toward charge exchange reactions $(\mathrm{Q} 1=-8 \mathrm{~V})$. charge exchange reaction

$$
\mathrm{Ar}^{+}+\mathrm{N}_{2} \rightarrow \mathrm{Ar}+\mathrm{N}_{2}^{+}
$$

has been studied extensively because of the proximity of the ionization potential of $\mathrm{N}_{2}$ and the ${ }^{2} P_{3 / 2}$ and ${ }^{2} P_{1 / 2}$ states of $\mathrm{Ar}^{+}$. Specifically, the charge transfer process is exoergic by 0.18 and $0.36 \mathrm{eV}$, respectively, for the two argon ion states. One might expect, therefore, that the reaction depicted in eq 5 would be quite facile in this system and that the $\mathrm{Ar}^{+}$attenuation factors would be more similar to those seen for the symmetric exchange case. Martinez and Dheandhanoo [21] in a discussion of the use of this reaction as part of a standardization procedure in the development of instrument-independent tandem mass spectrometry data bases, review previous work in characterizing this charge transfer process. In their concluding remarks the authors discuss the reasons why, in some instances, this reaction shows a very high cross section, whereas in other instances only moderate efficiency is observed. The disparity of published values $(2-3 \times)$ is far higher than those seen in many other reactions studied in different laboratories, which tend to vary by only $\pm 30 \%$. The variability in these values is due to the state-selective nature of the reaction (both of the primary ion and the resultant molecular ion) and its sensitivity to the energetics of the collision. Obviously, the collision parameters employed here do not favor the attenuation of $\mathrm{Ar}^{+}$via the reaction depicted in eq 5 .

Evidence for the asymmetric charge exchange between various discharge species and xenon target gas atoms was obtained and is illustrated in Figure 7. Based on the same energy selection theory stated previously with reference to Figure 6, the polarity of the bias voltage of the second quadrupole was varied to allow ions with different kinetic energies to be detected under different settings. Here, under typical quadrupole energy settings for $\mathrm{CID}(\mathrm{Q} 2=+18.6 \mathrm{~V})$, no secondary ions from charge exchange were seen, as indicated by the absence of $X_{e}$ in the $m / z \quad 124-136$ range. The $\mathrm{Sn}^{+}$peaks are due to the presence of tin in the brass sample employed in this study. As the quadrupole bias was set to more negative values, the instrument became capable of transmitting lower kinetic energy ions such as $\mathrm{Xe}^{+}$produced by charge exchange reactions. At $\mathrm{Q} 2=-3.9 \mathrm{~V}$, several $\mathrm{Xe}$ isotopes were observed, and this observation verifies their formation in the collision cell through asymmetric charge exchange reactions between $\mathrm{Ar}^{+}$and $\mathrm{Xe}$, or possibly $\mathrm{Ar}^{+2}$ and $\mathrm{Xe}$, to produce $\mathrm{Ar}^{+}$and $\mathrm{Xe}^{+}$[22]. The same type of charge exchange process(es) has also been demonstrated by Rowan and Houk [7], who used methane and xenon as target gases in ICP-MS.

\section{Conclusions}

Comparison of different target gases for collisioninduced dissociation is difficult due to the inability to 


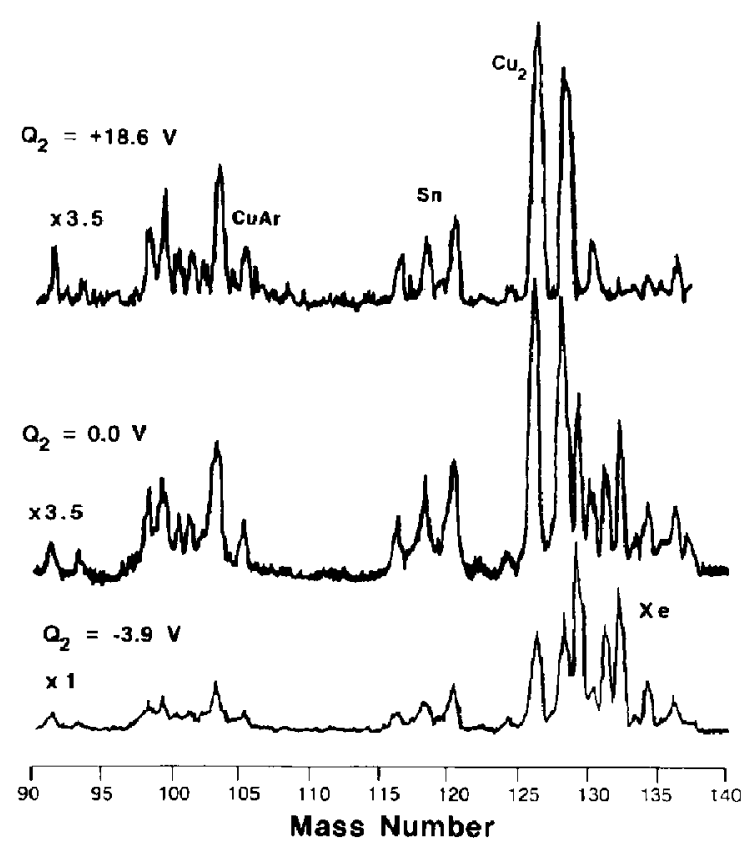

Figure 7. Evidence of charge exchange to xenon target gas atoms as a function of the analyzer quadrupole $(Q 2)$ bias potential.

discern mechanisms of loss of polyatomic species. Optimally, a triple quadrupole mass spectrometer would permit unequivocal identification of reaction pathways. The role of target mass and ionization potential provides indications of the relative contribution of CID, charge exchange, and scattering to such losses. Each of the investigated gases affects the preferential removal of polyatomic ions to some degree. Discharge gas ions with high ionization potentials are reduced (via charge exchange) relative to atomic analyte ions that have lower ionization potentials. Such preferential losses occur with varying degrees of success. Target gases with low ionization potentials, such as xenon, are favorable for charge exchange with monatomic species and perhaps polyatomic species as well. Charge exchange is of course most efficiently induced with like targets (i.e., symmetric charge exchange).

Argon typically has been used in the studies conducted to date by the authors. Of the alternative targets compared in this study, nitrogen is a reasonable candidate as a collision target because it has slightly better CID efficiencies than argon and somewhat lower scattering of the analyte, although the loss of $\mathrm{Ar}^{+}$is nowhere near as efficient. Xenon, however, is deter- mined to be the most effective target gas and hence the best choice for a collision gas in double quadrupole mass spectrometry. Cost considerations in the use of Xe are not of great importance because flow rates of $<1 \mathrm{~mL} / \mathrm{min}$ are employed in this system. Future studies will concentrate more explicitly on the analytical evaluation of the double quadrupole approach for affecting loss of problematic polyatomic ions in $\mathbf{r f}$ GDMS, and possibly other sources applied in plasma source mass spectrometry.

\section{Acknowledgment}

This work was funded by the National Science Foundation under grants CHE-8901788 and CHE-9117152.

\section{References}

1. Marcus, R. K., Ed. Glow Discharge Spectroscopies; Plenum: New York, 1993.

2. Harrison, W. W.; Bentz, B. L. Prog. Ahal. Spectrosc. 1988, 11, 53.

3. Duckworth, D. C.; Marcus, R. K. Anal. Chem. 1989, 61, 1879.

4. Duckworth, D. C.; Marcus, R. K. I. Anal. At. Spectrosc. 1992, 7, 711 .

5. King, F. L.; McCormack, A. L.; Harrison, W. W. I. Anal. At. Spectrom. 1988, 3, 883 .

6. Chapman, B. Glow Discharge Processes; Wiley-Interscience: New York, 1980.

7. Rowan, J. T.; Houk, R. S. Appl. Spectrosc. 1989, 43, 976.

8. King, F. L.; Harrison, W. W. Int. J. Mass Spectrom. Ion Processes $1989,89,171$.

9. Duckworth, D. C.; Marcus, R. K. Appl. Spectrosc. 1990, 44, 649.

10. Hu, K.; Houk, R. S. J. Am. Soc. Mass Spectrom. 1993, 4, 16.

11. McLuckey, S. A. J. Am. Soc. Mass Spectrom. 1992, 3, 599.

12. Chambers, D. M.; Hieftje, G. M. Spectrochim. Acta 1991, 46B, 761.

13. Olivares, J. A.; Houk, R. S. Appl. Spectrosc. 1985, 39, 1070.

14. Moseley, J. T.; Saxzon, R. P.; Huber, B. A.; Cosby, P. C.; Abouf, R.; Tadjeddine, M. J. Chem. Phys. 1977, 67, 1569.

15. Bauschlicher, Jr., C. W.; Partridge, H.; Langhoff, S. R. J. Chem. Phys. 1989, 91, 4733.

16. Lessen, D.; Brucat, P. J. Chem. Phys. Lett. 1988, 152, 473.

17. Lessen, D.; Brucat, P. J. J. Chem. Phys. 1989, 91, 4522.

18. Radzig, A. A.; Smirnov, B. M. Reference Data on Atoms, Molecules and Ions; Springer: Berlin, 1985; Chap. 11.

19. Dawson, P. H.; Douglas, D. J. In Tandem Mass Spectrometry; McLafferty, F. W., Ed.; Wiley Interscience: New York, 1983; Chap. 6.

20. Hergerburg, R.; Elford, M. T.; Skullerford, H. R. J. Phys. $B$ 1982, 15, 797.

21. Martinez, R. I.; Dheandhanoo, S. Int. J. Mass Spectrom. Ion Processes 1988, 84, 1 .

22. Smith, D.; Adams, N. G.; Alge, E.; Villinger, H.; Lindiger, W. J. Phys. B 1980, 13, 2787. 